\title{
Voevodsky's slice conjectures via Hilbert schemes
}

\author{
Tom Bachmann and Elden Elmanto
}

\begin{abstract}
We offer short and conceptual re-proofs of some conjectures of Voevodsky's on the slice filtration. The original proofs were due to Marc Levine using the homotopy coniveau tower. Our new proofs use very different methods, namely, recent development in motivic infinite loop space theory together with the birational geometry of Hilbert schemes.
\end{abstract}

\section{Introduction}

One major application of motivic homotopy theory is Voevodsky's construction of the AtiyahHirzebruch spectral sequence from motivic cohomology (which coincides Bloch's higher Chow groups [Blo86] of algebraic cycles up to reindexing [Voe02a]) converging to algebraic $K$-theory. While other constructions of this spectral sequence were proposed prior to motivic homotopy theory (notably [FS02, Lev01]), Voevodsky's approach is arguably the cleanest and most definitivewe refer to [Lev18, §2] for a survey. This spectral sequence is obtained via the slice filtration constructed in [Voe02b, Voe02c], which is a functorial filtration that one associates to a motivic spectrum $E$ :

$$
\cdots \rightarrow f_{q} E \rightarrow f_{q-1} E \rightarrow \cdots f_{0} E \rightarrow \cdots E
$$

The associated graded spectra are denoted by

$$
s_{q} E:=\operatorname{cofib}\left(f_{q+1} E \rightarrow f_{q} E\right)
$$

and are called the $q$ th slice of $E$. Letting $E$ equal KGL, the motivic spectrum representing algebraic $K$-theory, one obtains the desired spectral sequence.

While the construction of this filtration is formal, the identification of the spectral sequence (in other words, the associated graded pieces) hinged on the next two conjectures. They were stated by Voevodsky [Voe02c] and proved by Levine [Lev08].

Conjecture 1 ([Lev08, Theorem 10.5.1], [Voe02c, Conjecture 2]). Let $k$ be a perfect field and $\mathbf{1}_{k}$ denote the motivic sphere spectrum. Then $s_{0} \mathbf{1}_{k}$ canonically identifies with the spectrum representing motivic cohomology.

Conjecture 2 ([Lev08, Theorem 9.0.3], [Voe02c, Conjecture 3]). Let $k$ be a perfect field. The functor

$$
\omega^{\infty}: \mathcal{S H}(k) \rightarrow \mathcal{S H}^{S^{1}}(k)
$$

respects the slice filtration.

Received 12 March 2020, accepted in final form 14 March 2021.

2020 Mathematics Subject Classification 19E15, 14C35, 14F42.

Keywords: algebraic $K$-theory, motivic cohomology, motivic spectral sequence, framed correspondences, Hilbert schemes.

This journal is (C) Foundation Compositio Mathematica 2021. This article is distributed with Open Access under the terms of the Creative Commons Attribution Non-Commercial License, which permits non-commercial reuse, distribution, and reproduction in any medium, provided that the original work is properly cited. For commercial re-use, please contact the Foundation Compositio Mathematica. 


\section{Voevodsky's SLICE CONJECTURES VIA HILBERT SCHEMES}

We will recall the definition of the slice filtrations on $\mathcal{S H}(k)$ and $\mathcal{S H}^{S^{1}}(k)$ in the main text. Voevodsky further proved that the validity of Conjecture 2 ensures the convergence of the resulting spectral sequence [Voe02c, Corollary 3.4], while Conjecture 1 identifies the graded spectra as suspensions of the motivic cohomology spectrum [Voe02c, Section 5] based on periodicity properties of the motivic spectrum representing algebraic $K$-theory. As already mentioned in the first paragraph, these motivic cohomology spectra have concrete incarnations as Bloch's higher Chow groups, usually denoted by $C H^{i}(X, j)$, so that $C H^{i}(X, 0)$ is isomorphic to $C H^{i}(X)$, the Chow group of codimension $i$ algebraic cycles up to rational equivalence. In total, we obtain a strongly convergent, cohomologically-indexed spectral sequence

$$
E_{2}^{p, q}=H^{p-q}(X ; \mathbb{Z}(-q))=C H^{-q}(X,-p-q) \Rightarrow K_{-p-q}(X)
$$

whenever $X$ is a smooth scheme over a field.

The purpose of this paper is to give an independent, short, and conceptual proof of Conjecture 2 and a simplification of Levine's proof of Conjecture 1, assuming motivic infinite loop space theory $\left[\mathrm{EHK}^{+} 17, \mathrm{EHK}^{+}\right.$20, $\left.\mathrm{BEH}^{+} 21\right]$ as developed by the authors and Hoyois, Khan, Sosnilo, and Yakerson, building on foundational work of Ananyevskiy, Druzhinin, Garkusha, Neshitov, and Panin in the seminal papers [GP21, GP20, AGP21, GNP21, DP18], based on unpublished ideas of Voevodsky's. In particular, these papers gave rise to motivic infinite loop space theory by computing the infinite $\mathbb{P}^{1}$-loop space of a variety in terms of framed correspondences. In fact, a proof of Conjecture 2 along these lines was already envisioned by Voevodsky in [Voe02c], although our proof proceeds via rather different methods.

The proof of Conjecture 2 is independent because we make no reference to Levine's proof. It is short, given the length of this paper. Finally, it is conceptual because we can reformulate both conjectures as relatively elementary statements about the birational geometry of certain Hilbert schemes. Indeed, motivic infinite loop space theory furnishes for us geometric models for the infinite loop space of the motivic sphere spectrum and, in fact, the suspension spectrum of any smooth $k$-variety as framed Hilbert schemes $\left[\mathrm{EHK}^{+} 17, \S 5.1\right]$. That slices have something to do with the birational geometry of varieties is already well known in the literature [KS17, Pel14]. In lieu of proving Conjecture 1 as stated, we identify $s_{0}(\mathbf{1})$ with a certain explicit framed suspension spectrum. While this characterizes the spectrum uniquely, the relationship with higher Chow groups is not clear from this perspective. On the other hand, this simplifies the proof of Conjecture 1 by replacing Levine's use of his "reverse cycle map" with a Hilbert schemes argument; see Remark 16.

\section{Notation and conventions}

We fix a field $k$. We make use of the categories and functors depicted in the following diagram:

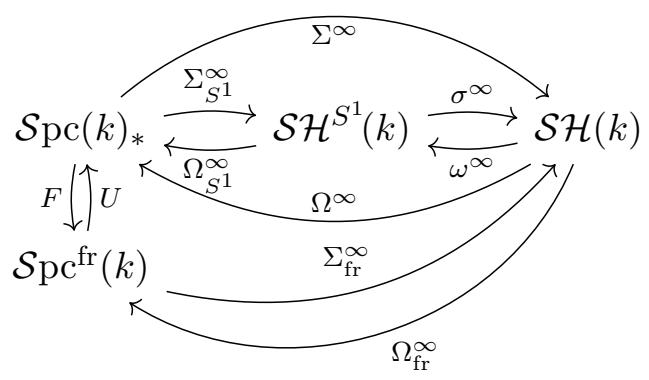




\section{T. BaChmann AND E. Elmanto}

Here $\mathcal{S p c}(k)_{*}, \mathcal{S} \mathcal{H}^{S^{1}}(k), \mathcal{S H}(k)$, and $\mathcal{S} \mathrm{pc}^{\mathrm{fr}}(k)$ denote, respectively, the pointed unstable motivic $\infty$-category (see, for example, [BH17, $\S 2.2]$ ), the category of $S^{1}$-spectra, that is, the stabilization of $\mathcal{S p c}(k)_{*}$, the category of motivic spectra (see, for example, [BH17, §4.1]), and the category of motivic spaces with framed transfers $\left[\mathrm{EHK}^{+} 17, \S 3.2\right]$. All parallel functors in opposite directions are adjoints, the functors $\Sigma^{\infty}, \Sigma_{S^{1}}^{\infty}, \sigma^{\infty}$ are the evident infinite suspension functors, and $U$ is the evident forgetful functor. The diagram of left adjoints (respectively, right adjoints) commutes.

We freely use the language of $\infty$-categories as set out in [Lur09, Lur16].

\section{Some birational geometry of framed Hilbert schemes}

For a scheme $X$ and a point $x \in X$, we write

$$
\operatorname{cod}_{X}(x)=\operatorname{dim}\left(\mathcal{O}_{X, x}\right)
$$

for the codimension of $x$ in $X$ (see also [Sta18, Tag 02IZ]). We will use several times the following well-known "codimension formula."

Theorem 3. Let $f: X \rightarrow Y$ be a flat morphism of locally noetherian schemes and $x \in X$. Then

$$
\operatorname{cod}_{X}(x)=\operatorname{cod}_{Y}(f(x))+\operatorname{cod}_{X_{f(x)}}(x) .
$$

Proof. Let $y=f(x)$. Note that $\mathcal{O}_{X_{y}, x}=\mathcal{O}_{X, x} \otimes_{\mathcal{O}_{Y, y}} k(y)$. The theorem is now a restatement of [GW10, Corollary 14.95].

For the rest of this section, we assume that all schemes are locally noetherian.

Definition 4. Let $d \in \mathbb{Z}$. An open immersion $U \hookrightarrow X$ is called $d$-birational if whenever $x \in X$ with $\operatorname{cod}_{X}(x) \leqslant d$, we have $x \in U$.

Example 5. If $d<0$, the condition is vacuous. If $d=0$, this coincides with what is usually called birational ( $U$ contains all generic points of $X)$.

Remark 6. It follows from [Sta18, Tag 02I4] that $d$-birational open immersions are stable under composition.

The codimension formula tells us that being a $d$-birational open immersion is fpqc local (on the base).

Corollary 7. Let $\alpha: U \rightarrow S$ be arbitrary and $p: Y \rightarrow S$ flat.

(1) If $\alpha$ is a $d$-birational open immersion, then so is the base change $\alpha_{Y}: U_{Y} \hookrightarrow Y$.

(2) If $p$ is surjective and $\alpha_{U}$ is a d-birational open immersion, then so is $\alpha$.

Proof. (1) Since open immersions are stable under base change, it is enough to show that for $y \in Y$ with $\operatorname{cod}_{Y}(y) \leqslant d$, we have $y \in U_{Y}$. By the codimension formula, we have $\operatorname{cod}_{Y}(y) \geqslant$ $\operatorname{cod}_{S}(p(y))$, whence $p(y) \in U$ by the $d$-birationality of $\alpha$. Thus $y \in U_{Y}$, as needed.

(2) The morphism $\alpha$ is an open immersion by faithfully flat descent [Sta18, Tag 02L3]. Let $s \in S$ with $\operatorname{cod}_{S}(s) \leqslant d$. Let $y \in Y_{s}$ be a generic point, so that $\operatorname{cod}_{Y_{s}}(y)=0$. Then by the codimension formula, we have $\operatorname{cod}_{Y}(y)=\operatorname{cod}_{S}(s) \leqslant d$, so that $y \in U_{Y}$. This implies $s \in U$, as needed.

Corollary 8. If $U \hookrightarrow X$ and $V \hookrightarrow Y$ are $d$-birational open immersions of flat $S$-schemes, then so is $U \times{ }_{S} V \hookrightarrow X \times{ }_{S} Y$. 


\section{Voevodsky's SLICE CONJECTURES VIA HILBERT SCHEMES}

Proof. We have the factorization

$$
U \times_{S} V \hookrightarrow U \times_{S} Y \hookrightarrow X \times_{S} Y
$$

in which both maps are $d$-birational open immersions by Corollary $7(1)$, and hence so is the composite by Remark 6 .

The next lemma furnishes a fiberwise criterion for being $d$-birational.

Lemma 9. Let $\alpha: U \hookrightarrow X$ be an open immersion of flat $S$-schemes. Then $\alpha$ is $d$-birational if and only if for every $s \in S$, the restriction $\alpha_{s}: U_{s} \hookrightarrow X_{s}$ is $\left(d-\operatorname{cod}_{S}(s)\right)$-birational.

Proof. Suppose that $\alpha$ is $d$-birational. Let $s \in S$ and $x \in X_{s}$ with $\operatorname{cod}_{X_{s}}(x) \leqslant d-\operatorname{cod}_{S}(s)$. By the codimension formula, we deduce

$$
\operatorname{cod}_{X}(x)=\operatorname{cod}_{S}(s)+\operatorname{cod}_{X_{s}}(x) \leqslant d,
$$

and hence $x \in U \cap X_{s}=U_{s}$.

Conversely, suppose that the fiberwise condition holds. Let $x \in X$ with $f(x)=s$, and suppose $\operatorname{cod}_{X}(x) \leqslant d$. Then by the codimension formula again, we have

$$
\operatorname{cod}_{X_{s}}(x)=\operatorname{cod}_{X}(x)-\operatorname{cod}_{S}(s) \leqslant d-\operatorname{cod}_{S}(s) .
$$

It follows that $x \in U_{s} \subset U$. This concludes the proof.

For a (finite locally free) morphism $p: S^{\prime} \rightarrow S$, we write $R_{p}$ for the Weil restriction functor [BLR90, Chapter 7].

Proposition 10. Let $p: S^{\prime} \rightarrow S$ be finite locally free and $X \rightarrow S^{\prime}$ smooth and quasi-projective. Let $\alpha: U \hookrightarrow X$ be a d-birational open immersion. Then $R_{p}(\alpha): R_{p}(U) \rightarrow R_{p}(X)$ is a d-birational open immersion.

Proof. Open immersions and smooth schemes are preserved under Weil restriction [BLR90, Proposition 7.6.2(i) and Proposition 7.6.5(h)]. In particular, $R_{p}(X) \rightarrow S$ is flat. Using Lemma 9, it is thus enough to show that for $s \in S$, the restriction $R_{p}(\alpha)_{s}$ is $\left(d-\operatorname{cod}_{S}(s)\right)$-birational. Let $s^{\prime} \in S_{s}$. Since $\operatorname{dim} S_{s}=0$, the codimension formula implies that $\operatorname{cod}_{S}(s)=\operatorname{cod}_{S^{\prime}}\left(s^{\prime}\right)$, and hence Lemma 9 implies that $\alpha_{s}$ is $\left(d-\operatorname{cod}_{S}(s)\right)$-birational. Since Weil restriction commutes with base change [CGP15, Proposition A.5.2(1)], we may thus assume that $S=\operatorname{Spec}(k)$ is the spectrum of a field. Applying Corollary 7(2), we may assume that $k$ is algebraically closed.

Writing $S^{\prime}$ as a finite disjoint union of its connected components and using Corollary 8, we may assume that $S^{\prime}$ is a finite local $k$-scheme, and so in particular $S^{\prime} \rightarrow S$ is a universal homeomorphism [Sta18, Tags 00J8 and 01S4]. We claim that the canonically induced square

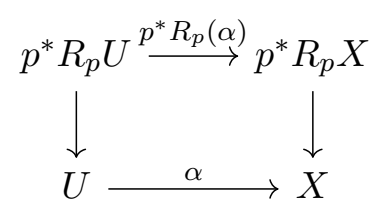

is cartesian. Indeed, for a scheme $T$ over $S^{\prime}$, maps into $p^{*} R_{p} X$ (over $S^{\prime}$ ) are the same as maps $T \times_{k}$ $S^{\prime} \rightarrow X$. We thus need to show that a map $T \times{ }_{k} S^{\prime} \rightarrow X$ factors through $U$ if and only if the composite $T \rightarrow T \times{ }_{k} S^{\prime} \rightarrow X$ does, which follows from the fact that the first map is a homeomorphism.

By [CGP15, Lemma A.5.11], the map $p^{*} R_{p} X \rightarrow X$ is faithfully flat, and hence $p^{*} R_{p}(\alpha)$ is $d$-birational by Corollary 7(1). Since $X \rightarrow S$ is faithfully flat, so is $p^{*} R_{p} X \rightarrow S$, and hence $R_{p}(\alpha)$ is $d$-birational by Corollary $7(2)$. 


\section{T. Bachmann and E. Elmanto}

With this preparation out of the way, we come to our main topic, Hilbert schemes. For $X \in$ $\mathrm{Sm}_{k}$ (quasi-projective, say), we write $\operatorname{Hilb}^{\mathrm{fr}}\left(\mathbb{A}^{n}, X\right)$ for the (ind-smooth) ind-scheme representing the functor $h^{\mathrm{nfr}, n}(X)$ of $\left[\mathrm{EHK}^{+} 17, \S 5.1 .4\right]$. We have

$$
\operatorname{Hilb}^{\text {fr }}\left(\mathbb{A}^{n}, X\right)=\coprod_{d \geqslant 0} \operatorname{Hilb}_{d}^{\text {frr }}\left(\mathbb{A}^{n}, X\right),
$$

the decomposition by degree; each $\operatorname{Hilb}_{d}^{\mathrm{fr}}\left(\mathbb{A}^{n}, X\right)$ is smooth. Finally,

$$
\operatorname{Hilb}^{\mathrm{fr}}\left(\mathbb{A}^{\infty}, X\right)=\underset{n}{\operatorname{colim}} \operatorname{Hilb}^{\mathrm{fr}}\left(\mathbb{A}^{n}, X\right)
$$

Lemma 11. If $\alpha: U \rightarrow X \in \mathrm{Sm}_{k}$ is a d-birational open immersion of smooth quasi-projective $k$-schemes, then so is $\operatorname{Hilb}_{m}^{\mathrm{fr}}\left(\mathbb{A}^{n}, U\right) \rightarrow \operatorname{Hilb}_{m}^{\mathrm{fr}}\left(\mathbb{A}^{n}, X\right)$.

Proof. There are maps $I \stackrel{q}{\rightarrow} Z \stackrel{p}{\rightarrow} \operatorname{Hilb}_{m}^{\text {ffci }}\left(\mathbb{A}^{n}\right)$ with $q$ smooth and $p$ finite locally free such that $\operatorname{Hilb}_{m}^{\mathrm{fr}}\left(\mathbb{A}^{n}, X\right) \cong R_{p}(I \times X)$; see $\left[\mathrm{EHK}^{+} 17, \S 5.1 .4\right]$. The result thus follows from Corollary 8 and Proposition 10 (using that $\operatorname{Hilb}_{m}^{\text {flci }}\left(\mathbb{A}^{n}\right)$ is smooth, and hence flat).

Recall that a $k$-scheme $X$ is rational if there exists a span of 0-birational open immersions $X \leftarrow U \rightarrow \mathbb{A}_{k}^{n}$ for some $n$.

Lemma 12. The scheme $\operatorname{Hilb}_{d}^{\mathrm{fr}}\left(\mathbb{A}^{n}, *\right)$ is rational.

Proof. We use the notation from the proof of Lemma 11. By construction, $I$ is a $\mathrm{GL}_{n}$-torsor over $Z$ (for the Zariski topology), and hence is birational to $\mathrm{GL}_{n} \times Z$, whence birational to $\mathbb{A}^{n^{2}} \times Z$. By the construction of the Weil restriction [BLR90, Theorem 7.6.4], the scheme $R_{p}\left(\mathbb{A}^{n^{2}} \times Z\right)$ is locally on $\mathrm{Hilb}^{\mathrm{flci}}\left(\mathbb{A}^{n}\right)$ isomorphic to a product with an affine space. Using Proposition 10, it is thus enough to show that $\operatorname{Hilb}_{d}^{\text {flci }}\left(\mathbb{A}^{n}\right)$ is rational. It is well known to be birational to $\operatorname{Sym}^{d}\left(\mathbb{A}^{n}\right)$ (see, for example, [Jel17, Lemma 4.28 and Theorem 4.36]), which is rational since $\mathbb{A}^{n}$ is [Mat68]. This concludes the proof.

\section{The birational localizations}

Denote by $L_{\text {bir }}^{d} \mathcal{S p c}(k)$ the (Bousfield) localization obtained by inverting $d$-birational open immersions of smooth $k$-schemes. See [Lur09, § 5.4.4] for one account on the localization of presentable $\infty$-categories. Variants of these localizations have been considered previously, for example by Kahn-Sujata [KS17] and Pelaez [Pel14].

Since $f \times \operatorname{id}_{X}$ is a $d$-birational open immersion whenever $f$ is (see, for example, Corollary 8), this is a symmetric monoidal localization (for example, apply [BH17, Proposition 6.16] to $S$ the spectrum of an algebraically closed field and $\mathcal{C}=\mathrm{FEt}_{S} \cong \mathrm{Fin}$ ). By Zariski descent, the same localization is obtained by inverting $d$-birational open immersions between smooth quasiprojective (or affine) $k$-schemes.

Write $L_{\text {bir }}^{d} \mathcal{S} \mathcal{H}^{S^{1}}(k)$ for the localization obtained by inverting maps of the form $\Sigma_{S^{1}}^{\infty+n} f_{+}$, with $n \in \mathbb{Z}$ and $f$ a $d$-birational open immersion. Similarly, write $L_{\text {bir }}^{d} \mathcal{S p c}(k)_{*}$ for the localization at maps of the form $f_{+}$, and $L_{\text {bir }}^{d} \mathcal{S} \mathrm{pc}^{\mathrm{fr}}(k)$ for the localization at maps of the form $F f$. These are also symmetric monoidal localizations.

Recall that $\mathcal{S H}^{S^{1}}(k)(d)$ is defined as the localizing subcategory generated by $\mathcal{S} \mathcal{H}^{S^{1}}(k) \wedge \mathbb{G}_{m}^{\wedge d}$. The reflection into its right orthogonal is denoted by $s_{[0, d-1]}$.

LemMa 13. Let $k$ be any field. 


\section{Voevodsky's SLICE CONJECTURES VIA HILBERT SCHEMES}

(1) The functors

$$
\mathcal{S p c}(k) \rightarrow \mathcal{S p c}(k)_{*} \rightarrow \mathcal{S H}^{S^{1}}(k)
$$

and $F: \mathcal{S p c}(k)_{*} \rightarrow \mathcal{S} \operatorname{pc}^{\text {fr }}(k)$ preserve $L_{\text {bir }}^{d}$-equivalences.

(2) The forgetful functor $\mathcal{S p c}(k)_{*} \rightarrow \mathcal{S} \mathrm{pc}(k)$ commutes with $L_{\text {bir }}^{d}$.

(3) $L_{\text {bir }}^{d}$-equivalences in $\mathcal{S p c}(k)_{*}$ are stable under finite products.

(4) The forgetful functor $U: \mathcal{S} \mathrm{pc}^{\mathrm{fr}}(k) \rightarrow \mathcal{S p c}(k)_{*}$ commutes with $L_{\text {bir }}^{d}$.

If $k$ is perfect, then the following also hold.

(5) A morphism $\alpha: E \rightarrow F \in \mathcal{S H} \mathcal{H}^{S^{1}}(k)$ is an $L_{\text {bir }}^{d}$-equivalence if and only if $\operatorname{cof}(\alpha) \in \mathcal{S} \mathcal{H}^{S^{1}}(k)(d+1)$. In fact, the localizing subcategory generated by objects of the form $\operatorname{cof}(\alpha)$, where $\alpha$ is an

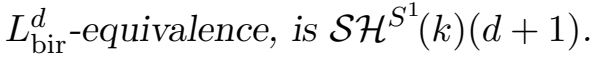

(6) For $E \in \mathcal{S H}^{S^{1}}(k)$, we have $L_{\text {bir }}^{d} E \simeq s_{[0, d]} E$.

In the proof, we shall make use of the theory of localizations of presentable $\infty$-categories and strongly saturated classes of morphisms [Lur09, § 5.5.4, Definition 5.5.4.5].

Proof. Assertion (1) is clear by construction, assertion (3) is immediate from assertion (2), and assertion (6) from assertion (5).

(2) By construction, the functor detects $L_{\text {bir }}^{d}$-local objects. This implies that it is enough to show that it preserves the strongly saturated class of morphisms (in $\left.\mathcal{S p c}(k)_{*}\right)$ generated by $d$-birational open immersions of smooth schemes. By [BH17, Lemma 2.10], for this it is enough to show that if $f$ is such a map and $X \in \operatorname{Sm}_{k}$, then $f \coprod \mathrm{id}_{X} \coprod \mathrm{id}_{*}$ is also a $d$-birational open immersion. This is clear.

(4) Using assertion (2), it suffices to show that the functor $\mathcal{S p c}^{\mathrm{fr}}(k) \rightarrow \mathcal{S}$ pc $(k)$ commutes with $L_{\text {bir }}^{d}$. Let us write $h^{\text {fr }}: \mathcal{S p c}(k) \rightarrow \mathcal{S p c}^{\text {fr }}(k)$ for the left adjoint of the previous functor; it is characterized as a sifted colimit-preserving functor which sends the (motivic localization of the) presheaf represented by smooth $k$-scheme $X$ to the (motivic localization of the) presheaf $h^{\text {fr }}(X)$ classifying tangentially framed correspondences (see $\left[\mathrm{EHK}^{+} 17\right.$, Definition 2.3.4] and below).

Arguing as above for assertion (2), we apply [BH17, Lemma 2.10] to $\mathcal{S} \mathrm{pc}^{\mathrm{fr}}(k)$. Using that $\operatorname{Spc}^{\text {fr }}(k)$ is semiadditive [EHK ${ }^{+} 17$, Proposition 3.2.10(iii)] and the localization $L_{\text {bir }}^{d}$ on $\mathcal{S p c}(k)$ is symmetric monoidal, it is enough to prove that if $f: X \rightarrow Y$ is a $d$-birational open immersion of smooth quasi-projective $k$-schemes, then $h^{\mathrm{fr}}(f): h^{\mathrm{fr}}(X) \rightarrow h^{\mathrm{fr}}(Y)$ becomes an equivalence in $L_{\text {bir }}^{d} \mathcal{S p c}(k)$. By [EHK ${ }^{+} 17$, Corollary 2.3.25 and Theorem 5.1.8], it is enough to show that $\operatorname{Hilb}^{\mathrm{fr}}\left(\mathbb{A}^{\infty}, X\right) \rightarrow \operatorname{Hilb}^{\mathrm{fr}}\left(\mathbb{A}^{\infty}, Y\right)$ is an $L_{\text {bir }}^{d}$-equivalence. This follows from Lemma 11.

(5) Using Lemma 14 below, it suffices to show the "in fact" part. Since $\alpha: X \times\left(\mathbb{A}^{n} \backslash 0\right) \rightarrow$ $X \times \mathbb{A}^{n}$ is $(n-1)$-birational and $\operatorname{cof}(\alpha) \simeq X_{+} \wedge T^{n}$, one inclusion is clear; for the other one, it is enough to show that if $U \hookrightarrow X$ is a $d$-birational open immersion, then $X / U \in \mathcal{S} \mathcal{H}^{S^{1}}(k)(d+1)$. This is well known; we include the proof for the convenience of the reader. Let $Z=X \backslash U$; we shall prove the claim by induction on $\operatorname{dim} Z$. By generic smoothness [Sta18, Tag 0B8X], there exists a smooth dense open $U^{\prime} \subset Z$; let $Z^{\prime}=Z \backslash U^{\prime}$. The cofiber sequence

$$
X \backslash Z^{\prime} / U \backslash Z^{\prime} \rightarrow X / U \rightarrow X / X \backslash Z^{\prime}
$$

implies that it is enough to show that $X \backslash Z^{\prime} / U \backslash Z^{\prime}, X / X \backslash Z^{\prime} \in \mathcal{S H}^{S^{1}}(k)(d+1)$. For the former, this follows from homotopy purity [MV99, Theorem 3.2.23], for the latter it holds by induction. This concludes the proof. 


\section{T. Bachmann and E. Elmanto}

We made use of the following technical result, which is surely well known.

Lemma 14. Let $\mathcal{C}$ be a stable presentable $\infty$-category and $S$ a small set of morphisms in $\mathcal{C}$ closed under desuspension. Write $S^{0}$ for the localizing subcategory of $\mathcal{C}$ generated by cofibers of morphisms in $S$. Then the strong saturation of $S$ consists of those maps $\alpha$ with $\operatorname{cof}(\alpha) \in S^{0}$.

Proof. Let $A$ be a strongly saturated class of morphisms stable under desuspensions, and write $A^{1}$ for the set of objects $X \in \mathcal{C}$ such that $0 \rightarrow X \in A$. Then $A^{1}$ is closed under colimits and desuspensions, hence is a localizing subcategory. Moreover by stability, $\alpha: X \rightarrow Y$ is an $A$-equivalence (that is, in $A$ ) if and only if $0 \rightarrow \operatorname{cof}(\alpha)$ is an $A$-equivalence. It follows that $A^{0}=A^{1}$, and also that $A \mapsto A^{0}$ is an inclusion-preserving bijection between strongly saturated classes stable under desuspension and localizing subcategories. Consequently, strongly saturated classes stable under desuspension containing $S$ are in bijection with localizing subcategories containing $S^{0}$. The result now follows from the observation that if $S$ is closed under desuspension, then so is its strong saturation.

As usual, we denote by $\mathcal{S H}(k)^{\text {eff }} \subset \mathcal{S H}(k)$ the localizing subcategory generated by $\sigma^{\infty} \mathcal{S H}^{S^{1}}(k)$ and put $\mathcal{S H}(k)^{\text {eff }}(d)=\mathbb{G}_{m}^{\wedge d} \wedge \mathcal{S H}(k)^{\text {eff }}$; this is equivalently the localizing subcategory generated by $\sigma^{\infty} \mathcal{S} \mathcal{H}^{S^{1}}(k)(d)$. We put $L_{\text {bir }}=L_{\text {bir }}^{0}$.

\section{Proof of Conjecture 1}

Recall from $[$ Hoy $21, \S 4]$ the framed presheaf $\mathbb{Z} \in \mathcal{P}_{\Sigma}\left(\operatorname{Corr}^{\mathrm{fr}}(k)\right)$, and from $\left[\mathrm{EHK}^{+} 17, \mathrm{EHK}^{+} 20\right]$ the presheaf $\mathcal{F} \mathcal{S}_{\mathrm{yn}}{ }^{\mathrm{fr}} \simeq h^{\mathrm{fr}}(*)$. There is an evident "degree" map of framed presheaves $\mathcal{F} \mathcal{S} \mathrm{yn}^{\mathrm{fr}} \rightarrow \mathbb{Z}$, factoring in fact through an evident sub-presheaf $\mathbb{N}$.

ThEOREM 15. Let $k$ be any field.

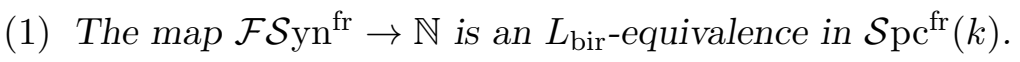

(2) The map $\Sigma_{\mathrm{fr}}^{\infty} \mathcal{F} \mathcal{S}_{\mathrm{yn}}{ }^{\mathrm{fr}} \rightarrow \Sigma_{\mathrm{fr}}^{\infty} \mathbb{Z}$ identifies with the canonical map

$$
\mathbf{1} \rightarrow s_{0}(\mathbf{1}) \in \mathcal{S H}(k) .
$$

Proof. (1) By Lemma 13(2,4), it suffices to show that the underlying map of unpointed motivic spaces is an $L_{\text {bir }}$-equivalence. Since it is the coproduct of the maps $\mathcal{F} \mathcal{S y n}{ }_{d}^{\text {fr }} \rightarrow *$ and $\mathcal{F} \mathcal{S y n}_{d}^{\text {fr }} \stackrel{L_{\text {mot }}}{\simeq}$ $\operatorname{Hilb}_{d}^{\mathrm{fr}}\left(\mathbb{A}^{\infty}, *\right)$, see $\left[\mathrm{EHK}^{+} 17\right.$, Corollary 2.3.25], it suffices to show that each $\operatorname{Hilb}_{d}^{\mathrm{fr}}\left(\mathbb{A}^{n}, *\right)$ is rational. This is Lemma 12.

(2) By [BH17, Lemma B.1] and [Hoy21, Lemma 20], all terms are stable under essentially smooth base change, so we may assume that $k$ is perfect. It follows from assertion (1) and Lemma 13(6) that $\mathbf{1} \simeq \Sigma_{\mathrm{fr}}^{\infty} \mathcal{F} \mathcal{S}_{\mathrm{yn}}{ }^{\mathrm{fr}} \rightarrow \Sigma_{\mathrm{fr}}^{\infty} \mathbb{N}$ induces an equivalence on $s_{0}$. Moreover, $\Sigma_{\mathrm{fr}}^{\infty} \mathbb{N} \simeq \Sigma_{\mathrm{fr}}^{\infty} \mathbb{Z}$ since $\mathbb{Z}$ is the group completion of $\mathbb{N}$. It thus remains to show that $\Sigma_{\mathrm{fr}}^{\infty} \mathbb{Z}$ is right orthogonal to $\mathcal{S H}(k)^{\mathrm{eff}}(1)$. But now, $\underline{\pi}_{*}\left(\Sigma_{\mathrm{fr}}^{\infty} \mathbb{Z}\right)_{0}$ is 0 if $* \neq 0$ and else $\mathbb{Z}$, so $\underline{\pi}_{*}\left(\Sigma_{\mathrm{fr}}^{\infty} \mathbb{Z}\right)_{-1}=0$, which implies what we want since homotopy sheaves are unramified. This concludes the proof.

Proof of Conjecture 1. The unit map $u: \mathbf{1} \rightarrow$ KGL induces a map $s_{0}(u): s_{0}(\mathbf{1}) \rightarrow s_{0}(\mathrm{KGL})$. Theorem 15(2), Conjecture 2 (to be proved in the next section), and Voevodsky's arguments from [Voe02c] (see also Example 22) imply that $s_{0}(u)$ is an equivalence. The zero slice of KGL is identified with higher Chow groups in [Lev08, Theorem 6.4.1], whence the result.

Remark 16. Theorem $15(2)$ shows that if $E \in \mathcal{S H}(k)^{\text {eff }}$ is provided with a map $\mathbf{1} \rightarrow E$ such that $\underline{\pi}_{i}(E)_{0}=0$ for $i \neq 0$ and $\underline{\pi}_{0}(\mathbf{1})_{0} \rightarrow \underline{\pi}_{0}(E)_{0}$ is isomorphic to $\underline{G W} \rightarrow \mathbb{Z}$, then the induced maps 


\section{Voevodsky's SLICE CONJECTURES VIA HILBERT SCHEMES}

$s_{0}(\mathbf{1}) \rightarrow s_{0}(E) \leftarrow E$ are both equivalences. Indeed, for the second equivalence, we just need to show that $f_{1} E \simeq 0$, which follows from $\underline{\pi}_{i}(E)_{-1}=0$ (since it is the contraction of the zero sheaf), and for the first equivalence, we need only verify that we get an isomorphism on $\underline{\pi}_{i}(-)_{0}$, which is now immediate from the theorem.

In principle, one can identify $s_{0}(\mathbf{1})$ with the spectrum representing higher Chow groups by verifying that the latter do satisfy these properties. However, at some point it needs to be proved that the spectrum representing higher Chow groups (even granting its existence) is effective. Voevodsky proves this (in characteristic zero) by studying the birational geometry of motivic Eilenberg MacLane spaces, and Levine deduces this from his homotopy coniveau tower theory. We have no new arguments for this. In effect, our argument replaces the deduction of $s_{0}(\mathbf{1})$ from $s_{0}(\mathrm{KGL})$ in $[\mathrm{Lev08}, \S 10]$ involving the "reverse cycle map."

\section{Proof of Conjecture 2}

For $d<0$, we define $\mathcal{S} \mathcal{H}^{S^{1}}(k)(d)=\mathcal{S} \mathcal{H}^{S^{1}}(k)$ and $f_{d}=\mathrm{id}: \mathcal{S H}^{S^{1}}(k) \rightarrow \mathcal{S} \mathcal{H}^{S^{1}}(k)(d)$. The next result is Conjecture 2. In Levine's approach [Lev08], he directly proves Corollary 19 below, which immediately implies the theorem.

TheOREM 17. Let $k$ be a perfect field. Then

$$
\omega^{\infty}\left(\mathcal{S H}(k)^{\mathrm{eff}}(d+1)\right) \subset \mathcal{S H}^{S^{1}}(k)(d+1) .
$$

Proof. If $d<0$, there is nothing to show. Otherwise, by Lemma 13(5), it suffices to show that if $f: X \rightarrow Y$ is a $d$-birational open immersion of smooth, quasi-projective $k$-schemes, then $\omega^{\infty} \Sigma_{+}^{\infty} f \in \mathcal{S H}^{S^{1}}(k)$ is an $L_{\text {bir }}^{d}$-equivalence. Using Lemma 18 below, this follows from Lemma $13(1,3,4)$.

The following technical result is a variant of [Voe02c, Proposition 4.4].

LEMma 18. Let $\mathcal{X} \in \mathcal{S p c}^{\mathrm{fr}}(k)$ (where $k$ is a perfect field). Then $\omega^{\infty} \Sigma_{\mathrm{fr}}^{\infty} \mathcal{X} \in \mathcal{S H}^{S^{1}}(k)$ can be obtained as a colimit of $S^{1}$-desuspensions of suspension spectra of the form $\Sigma_{S^{1}}^{\infty} U \mathcal{X}^{\times n}$. Moreover, this expression is natural in $\mathcal{X}$.

Proof. Writing $\omega^{\infty} \Sigma_{\mathrm{fr}}^{\infty} \mathcal{X}$ as the colimit of desuspensions of its constituent spaces, we have

$$
\omega^{\infty} \Sigma_{\mathrm{fr}}^{\infty} \mathcal{X} \simeq \operatorname{colim}_{n} \Sigma^{-n} \Sigma_{S^{1}}^{\infty} \Omega^{\infty} \Sigma^{n} \Sigma_{\mathrm{fr}}^{\infty} \mathcal{X}
$$

It is thus enough to prove that the spaces

$$
\Omega^{\infty} \Sigma_{\mathrm{fr}}^{\infty} \Sigma^{n} \mathcal{X} \in \mathcal{S p c}(k)_{*}
$$

are of the desired form, say for $n \geqslant 1$. We view $\mathcal{X}$ as an object of $\mathcal{P}_{\Sigma}\left(\operatorname{Corr}^{\mathrm{fr}}(k)\right)$. It follows from the motivic recognition principle $\left[\mathrm{EHK}^{+} 17\right.$, Theorem 3.5.14] that

$$
\Omega^{\infty} \Sigma_{\mathrm{fr}}^{\infty} \Sigma^{n} \mathcal{X} \simeq L_{\mathrm{mot}}^{\mathrm{gp}} \Sigma^{n} \mathcal{X}
$$

Writing $\Sigma^{n} \mathcal{X}$ as an iterated sifted colimit and using semiadditivity, we find that $\Sigma^{n} \mathcal{X} \simeq B^{n} \mathcal{X}$, where the bar construction $B^{n}$ is just applied sectionwise. In particular, $\Sigma^{n} \mathcal{X} \simeq B^{n} \mathcal{X}$ is of the desired form. Since the forgetful functor $\mathcal{P}_{\Sigma}\left(\operatorname{Corr}^{\mathrm{fr}}(k)\right) \rightarrow \mathcal{P}_{\Sigma}\left(\operatorname{Sm}_{k}\right)$ preserves motivic equivalences $\left[\mathrm{EHK}^{+} 17\right.$, Proposition 3.2.14], it suffices to show that $B^{n} \mathcal{X}$ is motivically group complete (that is, $L_{\text {mot }} B^{n} \mathcal{X}$ is group complete). Since $n \geqslant 1$, the presheaf $B^{n} \mathcal{X}$ is sectionwise connected (see, for example, [Seg74, Proposition 1.5]), and hence $L_{\text {mot }} B^{n} \mathcal{X}$ is connected by [MV99, Corollary 3.3.22]. This concludes the proof. 


\section{T. Bachmann and E. Elmanto}

We deduce the following structural result, also originally due to Levine [Lev08, Theorem 9.0.3]. Recall that $\mathcal{S H}(k) \simeq \mathcal{S} \mathcal{H}^{S^{1}}(k)\left[\mathbb{G}_{m}^{\wedge-1}\right]$, and so objects of $\mathcal{S H}(k)$ can be modeled by $\mathbb{G}_{m}$ - $\Omega$-spectra, that is, sequences $\left(E_{0}, E_{1}, \ldots\right)$ with $E_{i} \in \mathcal{S H}^{S^{1}}(k)$, together with equivalences $E_{i} \simeq \Omega_{\mathbb{G}_{m}} E_{i+1}$.

Corollary 19. (1) The functor $\omega^{\infty}: \mathcal{S H}(k) \rightarrow \mathcal{S H} \mathcal{H}^{S^{1}}(k)$ commutes with the functors $f_{d}$ and $s_{d}$ for all $d \in \mathbb{Z}$.

(2) If $E \in \mathcal{S H}(k)$ is represented by the $\mathbb{G}_{m}$ - $\Omega$-spectrum $\left(E_{0}, E_{1}, \ldots\right)$, then $f_{d} E$ is represented by the $\mathbb{G}_{m}-\Omega$-spectrum $\left(f_{d} E_{0}, f_{d+1} E_{1}, \ldots\right)$. In particular, $E$ is effective if and only if $E_{i} \in \mathcal{S H}^{S^{1}}(k)(i)$.

Proof. (1) The categories $\mathcal{S H}(k)^{\mathrm{eff}}(d)$ and $\mathcal{S} \mathcal{H}^{S^{1}}(k)(d)$ define non-negative parts of $t$-structures by [Lur16, Proposition 1.4.4.11]. By construction, $\sigma^{\infty}$ is right-t-exact, and hence $\omega^{\infty}$ is left$t$-exact. Since it is also right- $t$-exact by Theorem 17 , we deduce that $\omega^{\infty}$ is $t$-exact, that is, commutes with $f_{d}$. The claim about $s_{d}$ follows immediately.

(2) We have $E_{i} \simeq \omega^{\infty}\left(\mathbb{G}_{m}^{\wedge i} \wedge E\right)$. Hence we compute

$$
f_{d}(E)_{i} \simeq \omega^{\infty}\left(\mathbb{G}_{m}^{\wedge i} \wedge f_{d} E\right) \simeq \omega^{\infty}\left(f_{d+i}\left(\mathbb{G}_{m}^{\wedge i} \wedge E\right)\right) \simeq f_{d+i}\left(\omega^{\infty}\left(\mathbb{G}_{m}^{\wedge i} \wedge E\right)\right) \simeq f_{d+i}\left(E_{i}\right) .
$$

Here we have used part (1) for the third equivalence (which is the only non-trivial one in the string above). This concludes the proof.

We also deduce the following principle, slightly generalizing an argument of Voevodsky. The analog in topology is the following fact: if $E$ is a connective spectrum, then it is $d$-connective as soon as its infinite loop space is $d$-connective.

Proposition 20. Let $k$ be a perfect field and $E \in \mathcal{S H}(k)^{\mathrm{veff}}$. Then $E \in \mathcal{S H}(k)^{\mathrm{eff}}(d)$ as soon as $\Sigma^{\infty} \Omega^{\infty} E \in \mathcal{S H}(k)^{\mathrm{eff}}(d)$.

Proof. We may assume $d \geqslant 0$. Using the recognition principle and Lemma 18, or alternatively [Voe02c, Proposition 4.4], we find that $\omega^{\infty} E$ is in the localizing subcategory generated by suspension spectra of products of $\Omega^{\infty} E$. Since [Mor04, Lemma 6.2.2 and footnote 45]

$$
\Sigma_{S^{1}}^{\infty}(X \times Y) \simeq \Sigma_{S^{1}}^{\infty} X \vee \Sigma_{S^{1}}^{\infty} Y \vee \Sigma_{S^{1}}^{\infty} X \wedge Y
$$

this is equivalently the localizing subcategory generated by smash powers of $\Sigma_{S^{1}}^{\infty} \Omega^{\infty} E$. It follows that $\sigma^{\infty} \omega^{\infty} E$ is in the localizing subcategory generated by smash powers of $\Sigma^{\infty} \Omega^{\infty} E$, and hence (1) $\sigma^{\infty} \omega^{\infty} E \in \mathcal{S H}(k)^{\text {eff }}(d)$. By the triangle identities, the composite

$$
\omega^{\infty} E \rightarrow \omega^{\infty} \sigma^{\infty} \omega^{\infty} E \rightarrow \omega^{\infty} E
$$

is the identity, whence (2) $\omega^{\infty} E$ is a summand of $\omega^{\infty} \sigma^{\infty} \omega^{\infty} E$.

Corollary 19 implies that $F \in \mathcal{S H}(k)^{\text {eff }}$ is $d$-effective if and only if $\omega^{\infty} F$ is $d$-effective. Thus $\omega^{\infty} \sigma^{\infty} \omega^{\infty} E$ is $d$-effective by point (1), whence so is $\omega^{\infty} E$ by point (2), and hence so is $E$. This concludes the proof.

Example 21. We can use this to give a slightly different proof of Theorem 15 (that is, determine $\left.s_{0}(\mathbf{1})\right)$ over perfect fields. Namely, let $F$ denote the fiber of the degree map $\mathcal{F} \mathcal{S y n}^{\text {fr,gp }} \rightarrow \mathbb{Z}$. It is enough to show that $\Sigma^{\infty} F \in \mathcal{S H}(k)^{\mathrm{eff}}(1)$. But by $\left[\mathrm{BEH}^{+} 21\right]$, we have

$$
F \stackrel{L_{\operatorname{mot}}}{\simeq} \mathcal{F} \mathcal{S}_{\mathrm{yn}}^{\mathrm{fr}+} \stackrel{L_{\mathrm{mot}}}{\simeq} \operatorname{Hilb}_{\infty}^{\mathrm{fr}}\left(\mathbb{A}^{\infty}, *\right)^{+} .
$$

Since $\Sigma^{\infty}$ inverts acyclic maps, the result thus follows again from Lemma 12. 


\section{Voevodsky's SLICE CONJECTURES VIA HILBERT SCHEMES}

Example 22. The argument of Example 21 is modeled on Voevodsky's determination of $s_{0}(\mathrm{KGL})$, which we can restate in our language as follows: Using that $\Omega^{\infty} \mathrm{KGL}$ equals $\mathbb{Z} \times \mathrm{Gr}$, where $\mathrm{Gr}$ is the infinite Grassmannian variety, arguing as above, one is reduced to showing that Gr is rational. This is well known.

Example 23. The converse of Proposition 20 is false. Let $E=H \mathbb{Z} / 2 \wedge \mathbb{G}_{m}$. We claim that $\Sigma^{\infty} \Omega^{\infty} E \notin \mathcal{S H}(k)^{\text {eff }}(1)$. For this it suffices to construct a non-zero map $\Sigma^{\infty} \Omega^{\infty} E \rightarrow \Sigma H \mathbb{Z} / 2$ or, equivalently, a non-zero map $\Omega^{\infty} H \mathbb{Z} / 2 \wedge \mathbb{G}_{m} \rightarrow \Omega^{\infty} \Sigma H \mathbb{Z} / 2$. Any group-like monoid $M$ is equivalent as a pointed space to $\pi_{0}(M) \times M_{\geqslant 1}$; applying this construction sectionwise and projecting to the $M_{\geqslant 1}$ part, we obtain the desired non-zero map

$$
\Omega^{\infty} H \mathbb{Z} / 2 \wedge \mathbb{G}_{m} \rightarrow \Omega^{\infty}\left(H \mathbb{Z} / 2 \wedge \mathbb{G}_{m}\right)_{\geqslant 1} \simeq K(\mathbb{Z} / 2,1) \simeq \Omega^{\infty} \Sigma H \mathbb{Z} / 2
$$

\section{ACKNOWLEDGEMENTS}

We would like to acknowledge the influence of Marc Hoyois and Marc Levine on this paper and our education. We thank Maria Yakerson and an anonymous referee for comments on a draft.

\section{REFERENCES}

AGP21 A. Ananyevskiy, G. Garkusha, and I. Panin, Cancellation theorem for framed motives of algebraic varieties, Adv. Math. 383 (2021), 107681; doi:10.1016/j.aim.2021.107681.

$\mathrm{BEH}^{+} 21$ T. Bachmann, E. Elmanto, M. Hoyois, A. A. Khan, V. Sosnilo, and M. Yakerson, On the infinite loop spaces of algebraic cobordism and the motivic sphere, Epi Géom. Algéb. 5 (2021); doi: 10.46298/epiga.2021.volume5.6581.

BH17 T. Bachmann and M. Hoyois, Norms in motivic homotopy theory, Astérisque, to appear, arXiv: 1711.03061.

Blo86 S. Bloch, Algebraic cycles and higher K-theory, Adv. Math. 61 (1986), no. 3, 267-304; doi: 10.1016/0001-8708(86) 90081-2.

BLR90 S. Bosch, W. Lütkebohmert, and M. Raynaud, Néron models, Ergeb. Math. Grenzgeb. (3), vol. 21 (Springer-Verlag, Berlin, 1990); doi:10.1007/978-3-642-51438-8.

CGP15 B. Conrad, O. Gabber, and G. Prasad, Pseudo-reductive groups, 2nd ed., New Math. Monogr., vol. 26 (Cambridge Univ. Press, Cambridge, 2015); doi:10.1017/CB09781316092439.

DP18 A. Druzhinin and I. Panin, Surjectivity of the etale excision map for homotopy invariant framed presheaves, 2018, arXiv: 1808.07765.

EHK $^{+} 17$ E. Elmanto, M. Hoyois, A A. Khan, V. Sosnilo, and M. Yakerson, Motivic infinite loop spaces, Camb. J. Math., to appear, arXiv:1711.05248.

EHK $^{+} 20$, Modules over algebraic cobordism, Forum Math. Pi 8 (2020), e14; doi:10.1017/fmp. 2020.13.

FS02 E. M. Friedlander and A. Suslin, The spectral sequence relating algebraic $K$-theory to motivic cohomology, Ann. Sci. École Norm. Sup. (4) 35 (2002), no. 6, 773-875; doi:10.1016/ S0012-9593(02)01109-6.

GNP21 G. Garkusha, A. Neshitov, and I. Panin, Framed motives of relative motivic spheres, Trans. Amer. Math. Soc., appeared online on 27 April 2021, to appear in print; doi:10.1090/tran/ 8386.

GP20 G. Garkusha and I. Panin, Homotopy invariant presheaves with framed transfers, Camb. J. Math. 8 (2020), no. 1, 1-94; doi:10.4310/CJM.2020.v8.n1.a1.

GP21 Framed motives of algebraic varieties (after V. Voevodsky), J. Amer. Math. Soc. 34 (2021), no. 1, 261-313; doi:10.1090/jams/958. 


\section{T. Bachmann and E. Elmanto}

GW10 U. Görtz and T. Wedhorn, Algebraic geometry I. Schemes with examples and exercises, Adv. Lect. Math. (Vieweg + Teubner, Wiesbaden, 2010); doi:10.1007/978-3-8348-9722-0.

Hoy21 M. Hoyois, The localization theorem for framed motivic spaces, Compos. Math. 157 (2021), no. $1,1-11$; doi:10.1112/s0010437x20007575.

Jel17 J. Jelisiejew, Hilbert schemes of points and their applications, Ph.D. Thesis, 2017, University of Warsaw, available at https://www.mimuw.edu.pl/sites/default/files/jjelisiejew_phd. pdf.

KS17 B. Kahn and R. Sujatha, Birational motives, II: Triangulated birational motives, Int. Math. Res. Not. 2017 (2017), no. 22, 6778-6831; doi:10.1093/imrn/rnw184.

Lev01 M. Levine, Techniques of localization in the theory of algebraic cycles, J. Algebraic Geom. 10 (2001), no. 2, 299-363.

Lev08 The homotopy coniveau tower, J. Topol. 1 (2008), no. 1, 217-267; doi:10.1112/ jtopol/jtm004.

Lev18_, Vladimir Voevodsky - an appreciation, Bull. Amer. Math. Soc. (N.S.) 55 (2018), no. 4, 405-425; doi:10.1090/bull/1637.

Lur09 J. Lurie, Higher topos theory, Ann. of Math. Stud., vol. 170 (Princeton Univ. Press, Princeton, NJ, 2009); doi:10.1515/9781400830558.

Lur16 Higher algebra, 2016, available at http://www.math.harvard.edu/ lurie/papers/ HA.pdf.

Mat68 A. Mattuck, The field of multisymmetric functions, Proc. Amer. Math. Soc. 19 (1968), 764-765; doi: $10.2307 / 2035879$.

Mor04 F. Morel, An introduction to $\mathbb{A}^{1}$-homotopy theory, in Contemporary Developments in Algebraic K-Theory, ICTP Lect. Notes, vol. 15 (Abdus Salam Int. Cent. Theoret. Phys., Trieste, 2004), 357-441.

MV99 F. Morel and V. Voevodsky, $\mathbf{A}^{1}$-homotopy theory of schemes, Publ. Math. Inst. Hautes Études Sci. (1999), no. 90, 45-143; doi:10.1007/BF02698831.

Pel14 P. Pelaez, The unstable slice filtration, Trans. Amer. Math. Soc. 366 (2014), no. 11, 5991-6025; doi:10.1090/S0002-9947-2014-06116-3.

Seg74 G. Segal, Categories and cohomology theories, Topology 13 (1974), 293-312; doi:10.1016/ 0040-9383 (74) 90022-6.

Sta18 The Stacks Project Authors, Stacks Project, version 2018, available at https://stacks.math. columbia.edu/.

Voe02a V. Voevodsky, Motivic cohomology groups are isomorphic to higher Chow groups in any characteristic, Int. Math. Res. Not. 2002 (2002), no. 7, 351-355; doi:10.1155/S107379280210403X.

Voe02b_- Open problems in the motivic stable homotopy theory. I, Motives, Polylogarithms and Hodge Theory, Part I (Irvine, CA, 1998), Int. Press Lect. Ser., vol. 3 (Int. Press, Somerville, MA, 2002), 3-34.

Voe02c A possible new approach to the motivic spectral sequence for algebraic $K$-theory, Recent Progress in Homotopy Theory (Baltimore, MD, 2000), Contemp. Math., vol. 293 (Amer. Math. Soc., Providence, RI, 2002), 371-379; doi:10.1090/conm/293/04956.

Tom Bachmann tom.bachmann@zoho.com

Department Mathematik, LMU München, München, Germany

Elden Elmanto elmanto@math.harvard.edu

Department of Mathematics, Harvard University, Cambridge, USA 